\title{
Evaluation of Lymphocyte Transformation Test Results in Patients with Delayed Hypersensitivity Reactions following the Use of Anticonvulsant Drugs
}

\author{
Zahra Karami $^{\mathrm{a}}$ Mehrnaz Mesdaghi ${ }^{\mathrm{a}, \mathrm{c}, \mathrm{e}}$ Parvaneh Karimzadeh ${ }^{\mathrm{d}, \mathrm{f}}$ \\ Mahboubeh Mansouric Mohammad Mehdi Taghdiri ${ }^{d}$ f \\ Zarrintaj Kayhanidoost $^{9} \quad$ Bita Jebelli $^{\text {h }}$ Reza Shekarriz Foumani ${ }^{\text {b }}$ \\ Delara Babaie $^{c, e} \quad$ Zahra Chavoshzadeh ${ }^{c, e}$
}

\begin{abstract}
Departments of ${ }^{\mathrm{a}}$ Immunology and ${ }^{\mathrm{b}}$ Social Medicine, School of Medicine, Shahid Beheshti University of Medical Sciences, Departments of ${ }^{C}$ Immunology and Allergy and ${ }^{d}$ Pediatric Neurology, ${ }^{e}$ Pediatric Infections Research Center and f Pediatric Neurology Research Center, Mofid Children's Hospital, Shahid Beheshti University of Medical Sciences, ${ }^{9}$ Department of Pediatric Neurology, Imam Khomeini Hospital, Tehran University of Medical Sciences, and ${ }^{h}$ Department of Pediatric Neurology, Hazrate Rasool Hospital, Iran University of Medical Sciences, Tehran, Iran
\end{abstract}

\section{Key Words}

Lymphocyte transformation test · Hypersensitivity reaction · Anticonvulsant drugs · Phenobarbital · Phenytoin ·

Carbamazepine · Lamotrigine

\begin{abstract}
Background/Aim: Administration of the anticonvulsant drugs phenobarbital, phenytoin, carbamazepine and lamotrigine can be associated with severe hypersensitivity reactions. The lymphocyte transformation test (LTT) is a method to determine which drug has caused the hypersensitivity reaction. This study was done to evaluate the results of LTT in patients with delayed hypersensitivity reactions following the administration of anticonvulsants. Methods: Twentyfour patients with hypersensitivity reactions, e.g. drug-induced hypersensitivity syndrome/drug rash and eosinophilia with systemic symptoms (DIHS/DRESS), Stevens-Johnson
\end{abstract}

syndrome (SJS) and toxic epidermal necrosis (TEN), following the administration of anticonvulsant drugs, and 24 patients who had used anticonvulsant drugs but did not have hypersensitivity reactions (the control group) were included in this study. Peripheral blood mononuclear cells were isolated. The cells were stimulated with the drugs, phytohemagglutinin as a mitogen and Candida as an antigen (positive controls). Lymphocyte proliferation was measured using the BrdU proliferation assay kit (Roche, Germany). The stimulation index was calculated as the mean ratio of the OD of stimulated cells divided by the OD of unstimulated cells. The results in the case and control groups were compared. Results: Of 24 patients in the test group, 14 (58.3\%) had positive LTT results and $10(41.7 \%)$ had negative results. Among patients in the control group, 1 (4.2\%) had a positive LTT result and $23(95.8 \%)$ had negative results. Among the patients who had received carbamazepine and phenytoin, there was a significant difference between the results of LTT in the case and

\section{KARGER}

E-Mail karger@karger.com

www.karger.com/iaa (c) 2016 S. Karger AG, Basel

$1018-2438 / 16 / 1703-0158 \$ 39.50 / 0$
Correspondence to: Dr. Mehrnaz Mesdaghi

Department of Immunology and Allergy, Mofid Children's Hospital

Shahid Beheshti University of Medical Sciences

Tehran (Iran)

E-Mail m.mesdaghi@sbmu.ac.ir 
control groups ( $p=0.002$ and $p=0.028$, respectively). Although patients receiving lamotrigine and phenobarbital had more positive LTT results in the case group than in the control group, these differences were not statistically significant. The sensitivity, specificity, positive predictive value and negative predictive value of $L T T$ were $58.4,95.8,93.3$ and $69.9 \%$, respectively. Conclusions: Considering the significant difference in LTT results between the case and control groups in patients receiving carbamazepine and phenytoin, and not observing such a difference in patients receiving phenobarbital and lamotrigine, LTT results are more valuable for the diagnosis of hypersensitivity reactions following the administration of carbamazepine and phenytoin. The $\mathrm{LTT}$ has good specificity but low sensitivity for the diagnosis of drug hypersensitivity reactions.

(c) 2016 S. Karger AG, Basel

\section{Introduction}

Hypersensitivity reactions to drugs can cause a variety of skin diseases like maculopapular, bullous and pustular eruptions. In recent years, increasing evidence indicates the important role of $\mathrm{T}$ cells in these drug-induced skin diseases [1]. Immune reactions to small molecular compounds, such as drugs, can cause a variety of diseases involving the skin, liver, kidney and lungs. In many drug hypersensitivity reactions, drug-specific $\mathrm{CD} 4+$ and $\mathrm{CD} 8+$ $\mathrm{T}$ cells recognize drugs through their alpha/beta $\mathrm{T}$-cell receptors in an MHC-dependent way [2]. Drugs can stimulate $\mathrm{T}$ cells like haptens via binding to self-peptides or interact directly with certain T-cell receptors. The activation of drug-specific $\mathrm{T}$ cells is generally thought to play an essential role in mediating adverse drug reactions such as Stevens-Johnson syndrome (SJS), toxic epidermal necrosis (TEN) and drug-induced hypersensitivity syndrome/drug rash and eosinophilia with systemic symptoms (DIHS/DRESS) $[3,4]$. The presence of cytotoxic drug-specific T cells also orchestrates inflammatory skin reactions through the release and induction of various cytokines (i.e. IL-5, IL-6, TNF- $\alpha$ and IFN- $\gamma$ ) and chemokines (RANTES or IL-8). The increased expression of these mediators seems to contribute to the generation of tissue and blood eosinophilia, a hallmark of many druginduced allergic reactions. However, in acute generalized exanthematous pustulosis (a peculiar form of drug allergy), neutrophils represent the predominant cell type within pustules, probably due to their recruitment by IL8 -secreting drug-specific T cells and keratinocytes [1]. In clinical settings, the patch test and lymphocyte transfor- mation test (LTT) are often used for the diagnostic assessment of drug-specific T-cell responses [5-15]. Despite it being widely understood that false-negative LTT reactions are observed in the acute stage regardless of the type of drug, studies have shown that positive LTT reactions can be observed when tests are performed 5-8 weeks after the onset of the drug reaction [16]. The aim of this study was to evaluate the results of LTT in patients with hypersensitivity reactions following anticonvulsant drug (phenobarbital, phenytoin, carbamazepine and lamotrigine) usage.

\section{Methods}

Twenty-four patients with hypersensitivity reactions following the administration of anticonvulsant drugs and 24 patients who had used anticonvulsant drugs but did not have hypersensitivity reactions (controls) were included in this study (table 1).

According to the clinical presentation, the adverse drug reactions were divided into 3 groups: SJS/TEN $(n=11)$, DIHS/DRESS $(n=9)$ and maculopapular skin eruptions $(n=8)$. The clinical criteria used for the diagnosis of SJS were the presence of widespread erythematous macules or flat atypical targets and detachment of $<10 \%$ of the body surface area and for TEN, the presence of widespread erythematous macules or flat atypical targets and detachment of $>30 \%$ of the body surface area [11]. The diagnostic criteria for DIHS/DRESS were a high fever, a widespread maculopapular and/or diffuse erythematous eruption, lymphadenopathy, leukocytosis with atypical lymphocytosis and/or eosinophilia and liver dysfunction [12-15]. The LTT was performed in the recovery stage, at least 6 weeks after SJS/TEN, DIHS/DRESS and skin rashes.

\section{Culture Medium and Chemicals}

Culture medium consisted of of RPMI 1640 (Gibco, Germany) supplemented with $10 \%$ pooled heat-inactivated human blood type AB serum, HEPES buffer ( $25 \mathrm{~mm}$; Gibco, Germany) and Lglutamine (2 mM; Gibco).

\section{Lymphocyte Transformation Test}

The culprit drugs used for LTT were unmodified parent drug compounds that were dissolved in suitable solvents (RPMI 1640 for phenobarbital and lamotrigine, distilled water for phenytoin and methanol alcohol for carbamazepine) and then sonicated. Solutions were filtered and stored at $-20^{\circ} \mathrm{C}$.

Peripheral blood mononuclear cells were isolated from whole blood using Ficoll/Hypaque (Sigma-Aldrich, USA) gradient centrifugation. The drug cytotoxic level was determined as the drug dose that can inhibit phytohemagglutinin (PHA)-induced lymphocyte proliferation (data not shown), and the suitable dose was defined as the dose that can induce the most proliferation [9]. The titration of the drug doses to find the suitable dose for LTT is depicted in figure $1.200 \mu \mathrm{l}$ of cell suspensions $\left(10^{6}\right.$ cells $\left./ \mathrm{ml}\right)$ were added to each culture-plate well and the cells were stimulated with $50 \mu \mathrm{g} / \mathrm{ml}$ of phenytoin, $100 \mu \mathrm{g} / \mathrm{ml}$ each of phenobarbital and carbamazepine and $25 \mu \mathrm{g} / \mathrm{ml}$ of lamotrigine. The cells were also stimulated with PHA and Candida antigen (as positive controls) in 


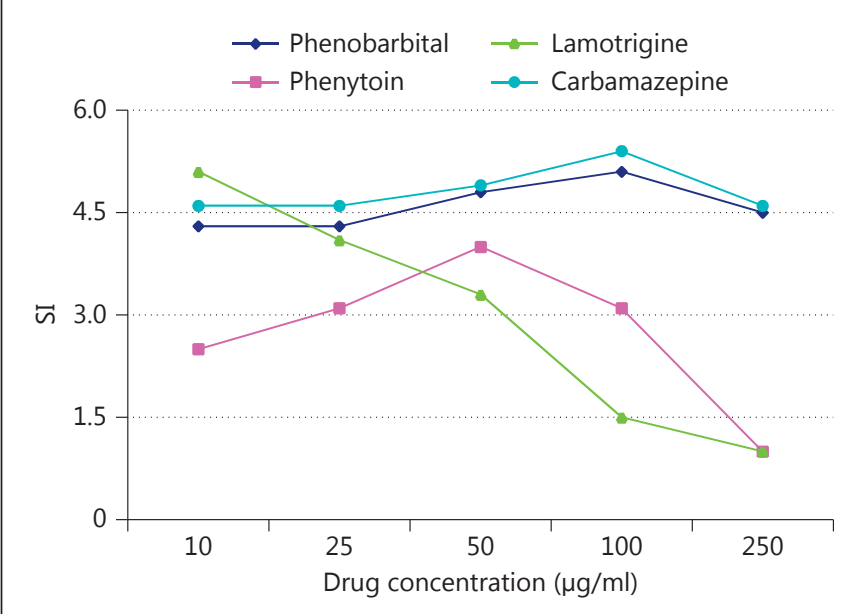

Fig. 1. Titration of drug doses to find the suitable dose for the LTT.

separate wells. The negative control comprised the cells without any stimulation. The cultures were performed in triplicate and incubated for 5 days in humidified $37^{\circ} \mathrm{C}$ incubator with $5 \% \mathrm{CO}_{2}$. Lymphocyte proliferation was measured, using a BrdU proliferation assay kit (Roche, Germany). The stimulation index (SI) was calculated as the mean ratio of the OD of stimulated cells divided by the OD of unstimulated cells. An SI $>2$ was considered as positive.

\section{Results}

Twenty-four patients ( 15 males and 9 females with a mean age of $13.66 \pm 6.02$ years) experiencing hypersensitivity reactions following the administration of anticonvulsant drugs, and 24 patients (15 males and 9 females with a mean age of $11.14 \pm 8.6$ years) who had used anticonvulsant drugs but not had hypersensitivity reactions, were included in this study, as the case and control groups, respectively. The clinical features and findings of these patients are summarized in table 1.

All patients had a normal response to PHA and Candida antigen (data not shown). Out of 24 patients in the case group, 14 (58.3\%) had positive LTT results and 10 $(41.7 \%)$ had negative test results. Among the patients in the control group, 1 (4.2\%) had a positive LTT result and $23(95.8 \%)$ had negative results. Among the patients who had received carbamazepine, 3 had a positive LTT result and 1 had a negative LTT result in the case group, but all 4 patients in the control group had a negative LTT result; this difference was statistically significant $(\mathrm{p}=0.028)$. Of the patients with hypersensitivity reactions following
Table 1. Characteristics of the patients

\begin{tabular}{lll}
\hline & Cases & Controls \\
\hline Mean age \pm SD years & $13.66 \pm 6.02$ & $11.14 \pm 8.6$ \\
Sex (male/female) & $15 / 9$ & $15 / 9$ \\
Drug & & \\
$\quad 10$ & 9 \\
$\quad$ Phenobarbital & 4 & 10 \\
$\quad$ Phenytoin & 4 & 4 \\
$\quad$ Carbamazepine & 8 & 2 \\
$\quad$ Lamotrigine & & \\
Type of drug reaction & 11 & \\
$\quad$ SJS/TEN & 9 & \\
DIHS/DRESS & 4 &
\end{tabular}

Values denote numbers of patients, unless otherwise specified.

Table 2. Types of drug reaction in patients with different drug usage

\begin{tabular}{llll}
\hline & SJS/TEN & DIHS/DRESS & $\begin{array}{l}\text { Maculopapular } \\
\text { eruptions }\end{array}$ \\
\hline Phenobarbital & 4 & 3 & 3 \\
Phenytoin & 3 & 1 & \\
Carbamazepine & 2 & 2 & 1 \\
Lamotrigine & 3 & 4 & \\
\hline
\end{tabular}

phenytoin administration, 3 had a positive LTT result and 1 had a negative result, but all the patients in the control group had a negative result $(p=0.002)$. LTT results were not significantly different between patients with and without hypersensitivity reactions following phenobarbital and lamotrigine usage (data not shown).

The mean \pm SD duration between the first intake of the drug and the onset of drug reactions was $65 \pm 50.7 \mathrm{~h}$. The mean time interval between drug intake and the development of a hypersensitivity reaction was $43.64 \pm 7.10 \mathrm{~h}$ in the case group with positive LTT results and $81.23 \pm 17.30$ $\mathrm{h}$ in the test group with negative test results. This difference was statistically significant $(\mathrm{p}=0.016)$.

Of 24 patients in the case group, $10(41.6 \%)$ took phenobarbital, $4(16.6 \%)$ had used phenytoin, $4(16.6 \%)$ took carbamazepine and 8 (33.3\%) took lamotrigine. The incidence of different types of drug reactions in patients using different drugs is shown in table 2 . There was no significant relationship between the drugs used and the type of drug reactions. 
Table 3. Relationship between LTT results and type of reaction

\begin{tabular}{lll}
\hline Reaction type & $\begin{array}{l}\text { Patients with } \\
\text { negative tests }\end{array}$ & $\begin{array}{l}\text { Patients with } \\
\text { positive tests }\end{array}$ \\
\hline SJS/TEN & $4(40)$ & $7(50)$ \\
DIHS/DRESS & $4(40)$ & $5(35.7)$ \\
Other & $2(20)$ & $2(14.3)$ \\
\hline Total & $10(100)$ & $14(100)$ \\
\hline \multicolumn{2}{c}{ Values denote $\mathrm{n}(\%)}$. \\
\hline
\end{tabular}

The mean duration of drug use in the control group that had negative LTT results was $135.04 \pm 30.45$ weeks, and in patients who had positive LTT results was 12 weeks. The differences between the duration of drug use and the LTT results were not statistically significant.

The incidence of different drug reactions in patients with positive and negative LTT results is summarized in table 3. No significant relationship was observed between LTT results and the type of drug reaction.

The mean \pm SD of the interval between the onset of the drug reactions and the time of performing LTT in the case group that had positive LTT results was $18 \pm 3.05$ months, and in patients who had negative LTT results, it was 35.23 \pm 7.52 months. The difference was statistically insignificant $(\mathrm{p}=0.174)$.

The sensitivity, specificity and positive and negative predictive values (PPV and NPV) were calculated. Fourteen patients in the case group had a positive LTT result and 10 had a negative result; 23 in the control group had a negative LTT and 1 had a positive LTT. According to the results of this study, LTT has a general sensitivity of $58.4 \%$ and specificity of $95.8 \%$ for determination of the drug that has caused drug hypersensitivity to anticonvulsants. LTT PPV for the diagnosis of drug hypersensitivity was $93.3 \%$ and its NPV was $69.9 \%$.

\section{Discussion}

One of the side effects of antiepileptic drugs is delayed hypersensitivity reactions. Anticonvulsant hypersensitivity syndrome is uncommon, but is potentially dangerous and life-threatening. The most common drugs that cause these reactions are phenytoin, phenobarbital and carbamazepine [17-20], and the most important reactions include TEN/SJS, DRESS and maculopapular eruptions that may lead to death $[20,21]$. Drug hypersensitiv- ity is one of the major problems among children and adults who are hospitalized and take different medications [22]. Early diagnosis of drug allergy syndrome and withdrawal of the culprit drug is helpful to prevent fatal results [23].

The most important and widely used tests for the diagnosis and choice of appropriate medications are the LTT and the skin patch test $[6,8,11-15,24]$. Activated T cells play an important role in drug reactions $[3,4,9,22]$. Kano et al. [16] showed that an SI $\geq 1.80$ can be regarded as positive, and Tang et al. [25] showed that an SI $\geq 2$ can be regarded as positive. In this study, we regarded SI $\geq 2$ as positive. Tang et al. [25] showed that the test should be performed during the recovery phase. In our study, LTT was done on all the patients at least 6 weeks after the last drug reaction.

We found that the LTT results were significantly different in the case and control groups in the patients who had used carbamazepine and phenytoin. A prospective study of 22 patients by Hari et al. [26] showed similar findings, with the LTT being positive in $67 \%$ of drug-allergic patients. In a cohort of carbamazepine-hypersensitive patients and controls, Naisbitt et al. [4] showed that the LTT was positive only in hypersensitive patients. The potential use of this test in a clinical situation requires further investigation. It might be helpful for the identification of the culprit drug in a patient on multiple drugs.

Considering significant differences between the case and control groups for patients receiving carbamazepine and phenytoin, and not observing such a difference in those receiving phenobarbital and lamotrigine, we conclude that the LTT results are more valuable for diagnosis of hypersensitivity following administration of carbamazepine and phenytoin.

In the study by Gex-Collet et al. [27], it was shown that LTT was often positive even years after the allergic reaction, indicating that the sensitization was persistent and detectable for a long time. The authors also stated that there was no significant relationship between the time interval, i.e. how long after the hypersensitivity reaction was performed, and the test results, and that this test can indeed be used for some years to find the culprit drug. In this study, we also showed no significant relationship between the time interval between the drug reaction and performing LTT and the results of the test.

In this study, 1 patient in the control group had a positive LTT result. Although several studies have reported that LTT can identify allergic subjects, nonallergic exposed controls can also respond. In the research by $\mathrm{Ny}-$ feler and Pichler [9], 2 of the nonatopic controls with es- 
tablished tolerance to $\beta$-lactams had a positive LTT result for benzylpenicillin and amoxicillin, respectively. This feature cannot be clearly explained.

The LTT has many advantages. This test is safe, it offers the possibility of investigating multiple drugs at the same time and its sensitivity and specificity are reported as $60-70 \%$ and $100 \%$, respectively, for anticonvulsant drugs [4]. In a retrospective study derived from the clinical history of 923 drug-allergic patients, the test was shown to have a sensitivity and specificity of $78 \%$ and $85 \%$, respectively [9]. According to the results of our study, the LTT has a general sensitivity of $58.4 \%$ and specificity of $95.8 \%$ for determining which drug has caused the hypersensitivity to anticonvulsants.

According to the results of different studies, including this one, the LTT has a high specificity but a limited sensitivity. A recent publication by Srinoulprasert and Pichler [28] showed that the depletion of regulatory T cells can enhance LTT sensitivity, without affecting specificity; this could be an important step toward the optimization of the LTT for the diagnosis of drug hypersensitivity reactions.

\section{References}

1 Pichler WJ, Yawalkar N, Britschgi M, Depta J, Strasser I, Schmid S, et al: Cellular and molecular pathophysiology of cutaneous drug reactions. Am J Clin Dermatol 2002;3:229-238.

2 Pichler WJ: Delayed drug hypersensitivity reactions. Ann Int Med 2003;139:683-693.

3 Pichler WJ, Tilch J: The lymphocyte transformation test in the diagnosis of drug hypersensitivity. Allergy 2004;59:809-820.

4 Naisbitt DJ, Britschgi M, Wong G, Farrell J, Depta JP, Chadwick DW, et al: Hypersensitivity reactions to carbamazepine: characterization of the specificity, phenotype, and cytokine profile of drug-specific T cell clones. Mol Pharmacol 2003;63:732-741.

5 Mori H, Yamanaka K, Kaketa M, Tamada K, Hakamada A, Isoda $\mathrm{K}$, et al: Drug eruption caused by azathioprine: value of using the drug-induced lymphocytes stimulation test for diagnosis. J Dermatol 2004;31:731-736.

6 Constable S, Farrell J, Naisbitt D, King C, Leonard N, Pirmohamed M: Systemic illness with skin eruption, fever and positive lymphocyte transformation test in a patient on irbesartan. Br J Dermatol 2006;155:491-493.

7 Buettiker U, Keller M, Pichler WJ, Braathen LR, Yawalkar N: Oral prednisolone induced acute generalized exanthematous pustulosis due to corticosteroids of group A confirmed by epicutaneous testing and lymphocyte transformation tests. Dermatology 2006;213:40-43.

8 Seishima M, Yamanaka S, Fujisawa T, Tohyama M, Hashimoto K: Reactivation of human herpesvirus (HHV) family members other than HHV-6 in drug-induced hypersensitivity syndrome. Br J Dermatol 2006;155:344-349.

9 Nyfeler B, Pichler WJ: The lymphocyte transformation test for the diagnosis of drug allergy: sensitivity and specificity. Clin Exp Allergy $1997 ; 27: 175-181$.

10 Luque I, Leyva L, Jose Torres M, Rosal M, Mayorga C, Segura JM, et al: In vitro T-cell responses to beta-lactam drugs in immediate and nonimmediate allergic reactions. Allergy 2001;56:611-618.
11 Bastuji-Garin S, Rzany B, Stern RS, Shear NH, Naldi L, Roujeau JC: Clinical classification of cases of toxic epidermal necrolysis, StevensJohnson syndrome, and erythema multiforme. Arch Dermatol 1993;129:92-96.

12 Shiohara T, Inaoka M, Kano Y: Drug-induced hypersensitivity syndrome (DIHS): a reaction induced by a complex interplay among herpesviruses and antiviral and antidrug immune responses. Allergol Int 2006;55:1-8.

13 Shiohara T, Kano Y: A complex interaction between drug allergy and viral infection. Clin Rev Allergy Immunol 2007;33:124-133.

14 Shiohara T, Iijima M, Ikezawa Z, Hashimoto $\mathrm{K}$ : The diagnosis of a DRESS syndrome has been sufficiently established on the basis of typical clinical features and viral reactivations. Br J Dermatol 2007;156:1083-1084.

15 Kano Y, Inaoka M, Shiohara T: Association between anticonvulsant hypersensitivity syndrome and human herpesvirus 6 reactivation and hypogammaglobulinemia. Arch Dermatol 2004;140:183-188.

16 Kano Y, Hirahara K, Mitsuyama Y, Takahashi $\mathrm{R}$, Shiohara T: Utility of the lymphocyte transformation test in the diagnosis of drug sensitivity: dependence on its timing and the type of drug eruption. Allergy 2007;62:14391444.

17 Seitz CS, Pfeuffer P, Raith P, Brocker EB, Trautmann A: Anticonvulsant hypersensitivity syndrome: cross-reactivity with tricyclic antidepressant agents. Ann Allergy Asthma Immunol 2006;97:698-702.

18 Kaur S, Sarkar R, Thami GP, Kanwar AJ: Anticonvulsant hypersensitivity syndrome. Pediatr Dermatol 2002;19:142-145.

19 Bavdekar SB, Muranjan MN, Gogtay NJ, Kantharia V, Kshirsagar NA: Anticonvulsant hypersensitivity syndrome: lymphocyte toxicity assay for the confirmation of diagnosis and risk assessment. Ann Pharmacother 2004;38: 1648-1650.
20 Mockenhaupt M, Messenheimer J, Tennis P, Schlingmann J: Risk of Stevens-Johnson syndrome and toxic epidermal necrolysis in new users of antiepileptics. Neurology 2005;64: 1134-1138.

21 Syn WK, Naisbitt DJ, Holt AP, Pirmohamed M, Mutimer DJ: Carbamazepine-induced acute liver failure as part of the DRESS syndrome. Int J Clin Pract 2005;59:988-991.

22 Thong BY, Mirakian R, Castells M, Pichler W, Romano A, Bonadonna P, et al: A World Allergy Organization international survey on diagnostic procedures and therapies in drug allergy/hypersensitivity. World Allergy Organ J 2011;4:257-270.

23 Armin S, Chavoshzadeh Z, Mohkam M, Rezaei N: Antiepileptic hypersensitivity and DRESS syndrome due to phenytoin in two pediatric cases. Turk J Pediatr 2009;51:76-77.

24 Kardaun SH, de Monchy JG: Acute generalized exanthematous pustulosis caused by morphine, confirmed by positive patch test and lymphocyte transformation test. J Am Acad Dermatol 2006;55(2 suppl):S21-S23.

25 Tang YH, Mockenhaupt M, Henry A, Bounoua M, Naldi L, Le Gouvello S, et al: Poor relevance of a lymphocyte proliferation assay in lamotrigine-induced Stevens-Johnson syndrome or toxic epidermal necrolysis. Clin Exp Allergy 2012;42:248-254.

26 Hari Y, Frutig-Schnyder K, Hurni M, Yawalkar N, Zanni MP, Schnyder B, et al: T cell involvement in cutaneous drug eruptions. Clin Exp Allergy 2001;31:1398-1408.

27 Gex-Collet C, Helbling A, Pichler WJ: Multiple drug hypersensitivity - proof of multiple drug hypersensitivity by patch and lymphocyte transformation tests. J Invest Allergol Clin Immunol 2005;15:293-296.

28 Srinoulprasert Y, Pichler WJ: Enhancement of drug-specific lymphocyte proliferation using $\mathrm{CD} 25^{\text {hi }}$-depleted $\mathrm{CD}^{+}$effector cells. Int Arch Allergy Immunol 2014;163:198-205. 